\title{
AN EXPERIMENTAL PLATFORM FOR THE ANALYSIS OF POLYDISPERSE SYSTEMS BASED ON LIGHT SCATTERING AND IMAGE PROCESSING
}

\author{
E. VIVIANI, F. ARMANI, A. BOSCOLO, B. PIUZZI, D. SALVALAGGIO \\ Department of Engineering and Architecture, University of Trieste, via Valerio 10, \\ 34127 Trieste, Italy \\ emanuele.viviani@phd.units.it
}

\begin{abstract}
In this work an experimental platform for light scattering analysis has been developed using image sensors, as CCD or CMOS. The main aim of this activity is the investigation of the feasibility of using these types of sensors for polydisperse systems analysis. The second purpose is the implementation of an experimental platform which is enough versatile to permit the observation of different phenomena in order to develop novel sensors/approach using data fusion.
\end{abstract}

\section{Introduction}

A polydisperse system is a mixture composed by a continue phase and two or more dispersed phases which have different characteristics like state of matter, shape, dimension and mass. Typical examples of this kind of systems are colloids and suspensions. Polydisperse systems can be found in different fields and their analysis is essential in a wide range of scientific and technological applications. For example in microbiology this type of analysis is used for the observation of microorganism behaviour and in biochemistry for the study of macromolecules. This method is widely used in the industry, both in synthesis and production processes, as pharmaceutical, cosmetic and detergent production processes.

One of the physical phenomena that can be used to investigate these systems is the light scattering. This has been modelled for the first time by Rayleigh and Mie, from the end of ' 800 and beginning of ' $900[1,2,3]$. Other models and different methods of analysis were developed for particles dispersed, like SLS (Static Light Scattering), DLS (Dynamic Light Scattering) and turbidimetry/nephelometry $[4,5]$. Various evolutions of these techniques have been developed recently allowing their use in new applications. The last years have been interested by a rapid development of new techniques like SALS (Small Angle Light Scattering), NFS (Near Field Scattering) and different type of cross-correlation methods [6,7].

Current solutions that can be found on the market are often expensive and are designed for laboratory or in-line application. These solutions aren't cost 
effective, thus they can't be applied in consumer products. However, there is the necessity of having low cost sensors that could provide some information about the characteristics of the dispersed particles. Moreover a first estimation of the properties of the polydisperse system could be sufficient in many applications, in which the accuracy requirements are weak. However often is not possible to discriminate between two system states when observing a single phenomenon.

The idea behind this work is to observe multiple phenomena involving light scattering and to apply data fusion to infer information about the system.

\section{Platform Architecture}

The composition of polydisperse systems influences several properties of scattered light: its intensity depends on wavelength and angle of observation; the dispersed particles could produce a polarization variation of scattered light or even a depolarization; movements of particles produce time variation behaviour of light intensity. Another effect that can be observed is the spatial distribution of scattered light when observing the phenomena with a camera.

In this work a video camera was chosen as the main sensor. This can be used in any of the aforementioned case. Some solutions are the use of external tools as polarization filters or multiple light sources of different wavelength.

In order to develop a versatile architecture, a classical goniometer-based setup was chosen for the platform [3, 4] (Figure 1).

The particular solution that was implemented consist of two concentric plates, one of which can rotate with an angular position actuator. Light sources and sensors can be mounted on both planes depending on which components need to be moved.

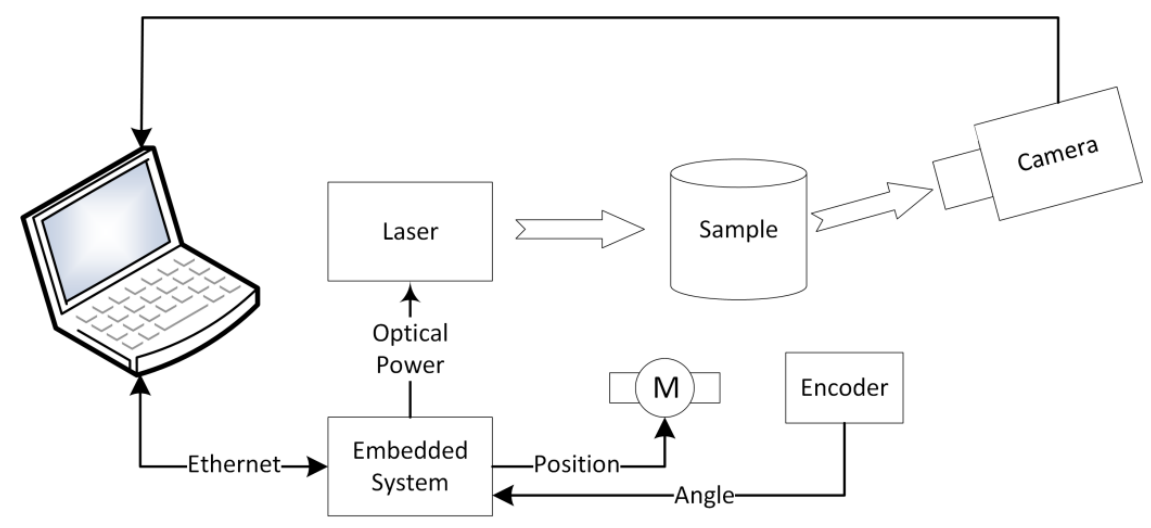

Figure 1 - Block diagram of platform architecture

A custom embedded electronic system control the platform. It was designed to have both digital and analog input-output capabilities. These allow managing more than one light source, like LEDs or laser diodes, and other optional light or temperature sensors. 
A computer manages the whole system, acquiring signals from camera and communicating over Ethernet with the embedded system.

The platform is entirely modular so that it can be customized depending on the test type for which it will be used. A dedicated software, can record both photo and video taken at fixed angle of view or in a continuous range. Moreover, it is capable of processing acquired images in real time or in a post processing step, even with the use of commercial image processing software.

The system has been calibrated using toluene as a reference. The detected scattered light has been used to normalize the response of the sensor. By this mean it was possible to compensate the intrinsic angular dependence of the goniometer-based setup [4].

\section{Preliminary Tests}

Some test were done in order to verify the observability of various phenomena with the developed platform.

Figure 2 shows two polar diagrams of different colloids which were obtained by averaging the light intensity signal of acquired images.

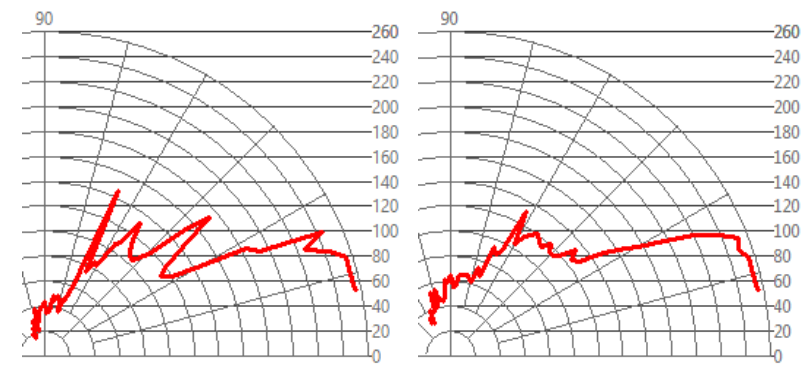

Figure 2 - Examples of light scattering polar diagram

In a second test, the use of a polarized filter was evaluated. This filter was placed in front of the camera, oriented orthogonal compared to the polarization of incident light. The test showed a good capability of the system to highlight the particles that induce light depolarization.

Figure 3 shows results of applying high-pass and low-pass spatial filters. With this processing example it is possible highlight either the particles or the background luminance. The latter component of the image is due to particles smaller than camera resolution. For example this techniques can be applied to improve signal to noise ration when one of the two components represent a noise for the measurement.

Additional tests were done in order to consider image processing that use time variations of the signal, like time averaging and time correlation. 
a)

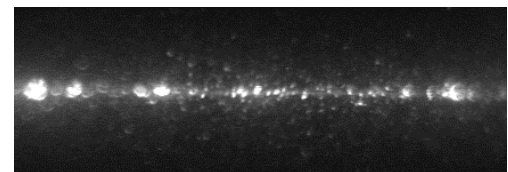

b)

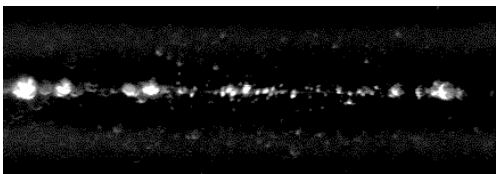

c)

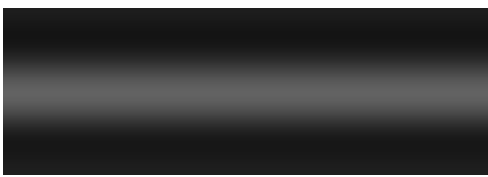

Figure 3 -a) Original acquired image; b) High-pass filtered image; c) Low-pass filtered image

\section{Conclusions}

The presented work represent a first step in the development of novel sensors for polydisperse systems analysis. On one hand the developed platform allows observing various phenomena; on the other it is a useful tool to test new techniques that merge different observed properties of scattered light. The use of a camera as a sensor allows the use of image processing tools. This will also enable the development of new analysis methods.

Future works will concern the metrological characterization of the system and the implementation of other type of processing. In a later stage a method to measure concentrations of components of a polydisperse system will be developed, in order to compare its performance to commercial solutions.

\section{References}

[1] H. C. Van de Hulst, Light Scattering by Small Particles, Courier Dover Publications, 1957.

[2] D. R. H. Craig F. Bohren, Absorption and Scattering of Light by Small Particles, Wiley, 1983.

[3] R. Xu, Particle characterization: light scattering methods, Professor Brian Scarlett, 2002.

[4] W. Schärtl, Light Scattering from Polymer Solutions and Nanoparticle Dispersions, Springer, 2006.

[5] M. Sadar, Turbidity Standards; Technical Information Series, Booklet 12; Hach Company: Loveland, CO, USA.

[6] F. Scheffold, R. Cerbino, New trends in light scattering, Current Opinion in Colloid \& Interface Science 12, 2007, 50-57.

[7] N. C. Alvarez, M. T. Garea, F. P. Quintian, Implementation and comparison of dynamic and static light scattering techniques for polydisperse particle sizing using a CCD camera, Journal of Physics: Conference Series 274, 2011. 